\title{
A preliminary study of the application of the transductal velocity ratio for assessing persistent ductus arteriosus
}

M W Davies, F R Betheras, M Swaminathan

\begin{abstract}
Objective-To compare the transductal velocity ratio (TVR) of the persistent ductus arteriosus (PDA) with other echocardiographic criteria for haemodynamic significance of a PDA.
\end{abstract}

Methods-This was a prospective study (from January 1997 to August 1998) in the nurseries of the Royal Women's Hospital, Melbourne. Infants with a clinically suspected PDA were eligible and included if the echocardiogram showed a PDA with a structurally normal heart and the TVR had been measured. The PDA was assessed for evidence of left heart dilatation, the presence of reverse or absent diastolic flow in the descending aorta, the pattern of Doppler flow velocity waveform in the ductus arteriosus, and subjective assessment of ductal diameter on the real time image. The peak systolic velocity (PSV) was obtained from the pulmonary and aortic ends of the PDA, and the TVR calculated by dividing the PSV at the pulmonary end by the PSV at the aortic end. Results-Forty two infants had 59 echocardiographs with their TVR calculated. Mean (SD) birth weight was 1008 (362) g. Mean (SD) gestational age at birth was 27.4 (2.2) weeks with a mean (SD) corrected gestational age of 28.7 (2.7) weeks. The mean TVR was decreased in those infants with a high left atrial diameter/aortic diameter (LA/Ao) ratio (1.9 $v 2.8, p=0.0032)$ or reverse/absent diastolic flow in the descending aorta $(2.1$ $v 3.0, \mathbf{p}=\mathbf{0 . 0 2}$ ). This difference was greater if those two criteria were combined $(1.7 \vee 3.4, \mathbf{p}=0.0027)$. The mean TVR was decreased in infants with a wide open duct seen on two dimensional imaging $(1.5 v 3.0, \mathbf{p}<0.0001)$ or pulsatile flow seen on pulsed Doppler in the PDA (1.9 v $3.4, p=0.0001)$. The LA/Ao and left ventricle internal diameter/aortic diameter (LVIDd/Ao) ratios were higher in the group with a TVR $<1.8$ than in the other two groups; these differences were statistically significant.

Conclusions-The TVR as a measure of the degree of constriction of a PDA is associated with other echocardiographic criteria for a haemodynamically significant PDA. A low TVR (signifying a poorly constricted duct) is associated with echocardiographic features of a significant left to right shunt, and vice versa.
Further research is required to determine the usefulness of the TVR in predicting closure or likely continuing patency of a PDA and the need for treatment.

(Arch Dis Child Fetal Neonatal Ed 2000;82:F195-F199)

Keywords: prematurity; persistent ductus arteriosus; echocardiography; transductal velocity ratio

Persistent ductus arteriosus (PDA) occurs commonly in preterm infants admitted to the neonatal intensive care unit. A PDA with significant left to right shunting can lead to complications such as respiratory distress, ${ }^{1}$ cardiac failure (partially compensated for by increased cardiac output), ${ }^{2}{ }^{3}$ low blood pressure, ${ }^{4}$ and decreased peripheral organ perfusion. ${ }^{2}$ A haemodynamically significant PDA leads to an increased incidence of intraventricular haemorrhage, ${ }^{5}$ necrotising enterocolitis, ${ }^{6}$ and chronic lung disease. ${ }^{78}$

The diagnosis of a PDA is easy to establish using current non-invasive echocardiographic techniques, especially colour flow Doppler. The important aspect of assessment of a PDA is whether the degree of left to right shunting across the PDA is of haemodynamic significance and will therefore require treatment. ${ }^{1}$

The increase in velocity of blood through a constricted PDA can be expressed as a ratio of the velocity at the pulmonary end of the duct to the velocity at the aortic end of the duct. This is the transductal velocity ratio (TVR) and it is proportional to the degree of constriction of the PDA. The calculation of such a velocity ratio is a standard method of assessing the degree of constriction in stenotic carotid arteries. ${ }^{910}$ The TVR has been used in our unit as a guide to the degree of constriction of a PDA; however, it has not been formally studied. The aim of this study was to compare the TVR with other echocardiographic criteria of haemodynamic significance.

\section{Methods}

This was a prospective study conducted, over a two year period from January 1997 to August 1998 , in the intensive and special care nurseries of the Royal Women's Hospital, Melbourne. Infants who had had an echocardiogram for a clinically suspected PDA were eligible to be studied. Those infants who had had their TVR measured (only if there was no right to left ductal flow), at the time of a cardiac scan were included in the study. Infants with any cardiac malformation (other than the PDA) or pulmonary hypertension were excluded. All echo- 
A

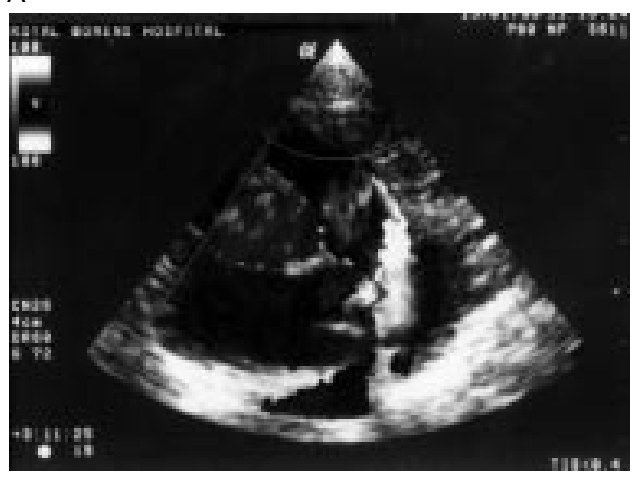

B

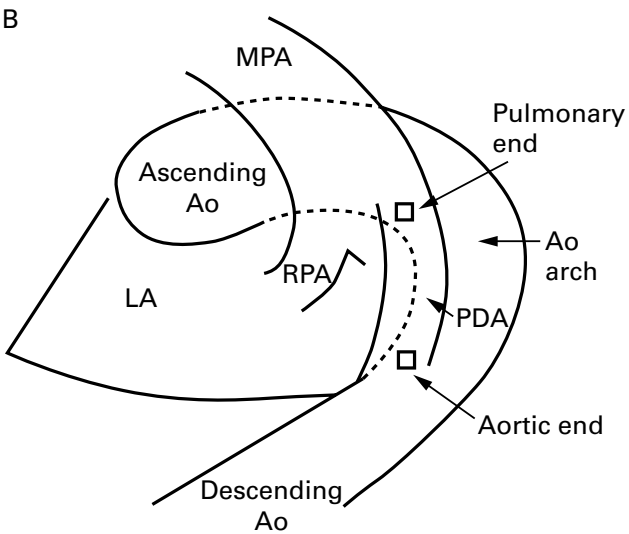

Figure 1 (A) Echocardiogram (with colour Doppler flow) showing the ductus arteriosus visualised in its longitudinal axis through an acoustic window at the left upper parasternal area. (B) Schematic diagram showing features shown in (A). The positioning of the pulsed Doppler sample volume is indicated by squares. MPA, main pulmonary artery; Asc Ao, ascending aorta; Pulm end, pulmonary end of the ductus arteriosus; Ao arch, aortic arch; PDA, persistent ductus arteriosus; Aortic end, aortic end of the ductus arteriosus; Desc Ao, descending aorta; LA, left atrium; $R P A$, right pulmonary artery.

cardiography was performed by a single operator (FRB).

The cardiac scans were performed using the S611 phased array sector probe $(5.7 \mathrm{MHz}$ for real time scanning and $4.0 \mathrm{MHz}$ for pulsed Doppler) on a GE LOGIQ 500 ultrasound scanner (GE Medical Systems, Waukesha, Wisconsin, USA). The standard acoustic windows and planes of scanning (apical four chamber view, left parasternal long axis and short axis views, subcostal view, right parasternal and suprasternal views) were used to obtain a complete assessment of cardiac anatomy, $M$

Table 1 Comparisons of mean (SD) transductal velocity ratios (TVR) between haemodynamically significant and non-significant ducts as assessed by various echocardiographic criteria

\begin{tabular}{lllc}
\hline Echocardiography criteria & Significant & Non-significant & $p$ Value \\
\hline $\begin{array}{l}\text { LA/Ao > 1.48 and reverse/absent } \\
\quad 1.7(0.8)(\mathrm{n}=18)\end{array}$ & $3.4(1.3)(\mathrm{n}=10)$ & 0.0027 \\
$\quad$ LAastolic flow & $1.9(0.9)(\mathrm{n}=26)$ & $2.8(1.2)(\mathrm{n}=31)$ & 0.0032 \\
LVIDd/Ao > 1.92 & $2.2(1.3)(\mathrm{n}=11)$ & $2.5(1.2)(\mathrm{n}=35)$ & 0.52 \\
Reverse/absent diastolic flow & $2.1(1.0)(\mathrm{n}=36)$ & $3.0(1.3)(\mathrm{n}=16)$ & 0.02 \\
Open on 2D echo image & $1.5(0.6)(\mathrm{n}=22)$ & $3.0(1.1)(\mathrm{n}=27)$ & $<0.0001$ \\
Pulsatile flow in PDA & $1.9(0.9)(\mathrm{n}=36)$ & $3.4(1.0)(\mathrm{n}=16)$ & 0.0001 \\
LVIDd > normal & $2.0(1.0)(\mathrm{n}=18)$ & $2.6(1.2)(\mathrm{n}=33)$ & 0.058 \\
\hline
\end{tabular}

Numbers in columns differ slightly due to unavailability of some variables in individual patients. $\mathrm{p}$ Values assessed by Student's $t$ test.

${ }^{\star}$ Reverse or absent diastolic flow in the descending aorta.

$\mathrm{LA} / \mathrm{Ao}$, left atrial diameter/aortic diameter ratio; LVIDd/Ao, left ventricle internal diameter/aortic diameter ratio. mode measurements of cardiac chambers and great vessels, and colour Doppler flow. The presence of a PDA was established using real time colour flow mapping and pulsed and/or continuous wave Doppler. The left atrial diameter/aortic diameter ratio (LA/Ao), left ventricular internal diameter in diastole (LVIDd), left ventricle internal diameter/aortic diameter ratio (LVIDd/Ao), and left ventricular fractional shortening were measured. Pulsed Doppler flow velocity waveforms in the descending aorta were assessed for the presence of reverse or absent diastolic flow. If possible, a two dimensional image of the PDA was obtained to determine if it was wide open or looked constricted. The pattern of Doppler flow velocity waveform in the ductus arteriosus was noted-that is, pulsatile or non-pulsatile. ${ }^{11}$ A LA/Ao ratio $>1.48$ and LVIDd/Ao ratio $>1.92$ were considered abnormal at any gestation; these numbers are derived from reference ranges developed in our unit (F R Betheras et al, unpublished data, 1998). The left ventricle was considered dilated if the LVIDd was outside our normal reference range for corrected gestational age ( $\mathrm{F} \mathrm{R}$ Betheras et al, unpublished data, 1998).

To obtain the TVR, the PDA was visualised in its longitudinal axis through an acoustic window at the left upper parasternal area. The entire PDA was seen in the centre of the image showing both the pulmonary and the aortic end clearly (fig 1). This plane was best obtained by using the colour flow mapping mode to visualise the ductal flow at its maximum intensity. Pulsed Doppler flow velocity waveforms were obtained from the pulmonary and aortic ends of the PDA, and the peak systolic velocity (PSV) measured. The TVR is derived by dividing the PSV at the pulmonary end by the PSV at the aortic end. Usually the colour mode was switched off to visualise the pulmonary and aortic ends of the duct clearly on two dimensional imaging for placement of the pulsed Doppler sample volume. The colour flow map of ductal flow usually extends beyond the anatomical edges of a duct and this can lead to incorrect placement of the sample volume.

The TVRs were divided into three groups for analysis: < 1.8, 1.8-3.7, and >3.7. These groups are used in assessment of the degree of carotid artery constriction, with systolic velocity ratios of $<1.8,1.8-3.7$, and $>3.7$ indicating $<60 \%, 60-79 \%$, and $\geqslant 80 \%$ diameter stenosis respectively. ${ }^{12}$

Non-skewed continuous data were analysed by Student's $t$ test or analysis of variance. Proportional data were analysed with the uncorrected $\chi^{2}$ test. Statistical significance was assumed at the 0.05 level.

\section{Results}

During the study period, 42 infants had 59 echocardiographs performed and had their TVR calculated. They had a mean (SD) birth weight of 1008 (362) g, and a mean (SD) gestational age at birth of 27.4 (2.2) completed weeks. At the time of the scan, their mean (SD) 
Table 2 LA/Ao ratio and aortic diastolic flow by the transductal velocity ratio of the patent ductus arteriosus

\begin{tabular}{|c|c|c|c|c|}
\hline & \multicolumn{4}{|c|}{ Transductal velocity ratio } \\
\hline & $<1.8$ & $1.8-3.7$ & $>3.7$ & $p$ Value \\
\hline Mean (SD) LA/Ao & $1.6(0.4)(\mathrm{n}=24)$ & $1.3(0.3)(\mathrm{n}=23)$ & $1.3(0.3)(\mathrm{n}=10)$ & $0.011^{\star}$ \\
\hline Mean (SD) LVIDd/Ao & $1.9(0.3)(\mathrm{n}=19)$ & $1.7(0.2)(\mathrm{n}=18)$ & $1.7(0.3)(\mathrm{n}=9)$ & $0.049^{\star}$ \\
\hline \multicolumn{5}{|l|}{ Number (\%) with } \\
\hline Forward diastolic flow & $4(17)$ & $7(35)$ & $5(56)$ & \\
\hline Reverse/absent diastolic flow & $19(83)$ & $13(65)$ & $4(44)$ & $0.096 \dagger$ \\
\hline
\end{tabular}

Numbers in columns differ slightly due to unavailability of some variables in individual patients.

$\star$ One way analysis of variance (ANOVA); $\dagger \chi^{2}$ test.

$\ddagger$ Reverse or absent diastolic flow in the descending aorta

LA/Ao, left atrial diameter/aortic diameter ratio; LVIDd/Ao, left ventricle internal diameter/aortic diameter ratio.

corrected gestational age was 28.7 (2.7) completed weeks, and their mean (SD) postnatal age was 10.7 (9.0) days.

Table 1 summarises differences in TVR between groups of infants with significant or non-significant PDAs (assessed by various echocardiographic criteria). The mean TVR was decreased in those infants with a high LA/Ao ratio or reverse/absent diastolic flow in the descending aorta. This difference was greater if these two criteria were combined; the group with both criteria present was compared with the group with both criteria absent. The mean TVR was decreased in those infants with a wide open duct seen on two dimensional imaging or pulsatile flow seen on pulsed Doppler in the PDA. All these differences were statistically significant. There was a trend towards a difference in mean TVR between groups with a dilated or non-dilated left ventricle, but this did not reach statistical significance. There was no difference in mean TVR when significance was defined as an increased LVIDd/Ao ratio.

Table 2 shows the differences in the degree of left heart overload and flow in the descending aorta by the TVR groups. The LA/Ao and LVIDd/Ao ratios were higher in the group with a TVR $<1.8$ ( $<60 \%$ stenosis) than in the other two groups. These differences are statistically significant. There were also more infants with reverse/absent aortic diastolic flow in this group, but this difference did not reach statistical significance.

To determine if the relation between TVR and haemodynamic significance holds true in those infants more likely to have higher pulmonary artery pressure, we analysed the data for infants $<5$ days old (table 3 ). The mean TVR was lower in infants with a high LA/Ao ratio or reverse/absent diastolic flow in the descending aorta; however, this difference was not statistically significant (this may be due to the small numbers of infants in this subgroup with a subsequent decrease in power to detect a

Table 3 Comparisons of mean (SD) transductal velocity ratios between haemodynamically significant and non-significant ducts as assessed by various echocardiographic criteria for infants $<5$ days old

\begin{tabular}{llll}
\hline $\begin{array}{l}\text { Echo criteria used to assess } \\
\text { significance of } P D A\end{array}$ & Significant & Non-significant & p Value \\
\hline LA/Ao $>1.48$ & $1.5(1.0)(\mathrm{n}=9)$ & $2.3(1.1)(\mathrm{n}=12)$ & 0.10 \\
Reverse/absent diastolic flow & $1.7(0.9)(\mathrm{n}=12)$ & $2.6(1.4)(\mathrm{n}=7)$ & 0.14
\end{tabular}

Numbers in columns differ slightly due to unavailability of some variables in individual patients. $\mathrm{p}$ Value assessed by Student's $t$ test.

* Reverse or absent diastolic flow in the descending aorta.

$\mathrm{LA} / \mathrm{Ao}$, left atrial diameter/aortic diameter ratio. significant difference if a difference truly exists).

To determine which PSV (pulmonary end or aortic end) contributes more to the TVR, the correlation coefficient $(r)$ between TVR and PSV was calculated for each: for TVR $v$ aortic end PSV, $r=0.18\left(r^{2}=0.034, p=0.91\right)$; for TVR $v$ pulmonary end PSV, $r=0.48$ $\left(r^{2}=0.235, \mathrm{p}=0.001\right)$. Most of the contribution toward TVR is from the pulmonary end PSV. However, when the same analyses as performed above for the TVR (as in table 1) were carried out with the pulmonary end PSV, we found no significant differences in the variables studied.

\section{Discussion}

Given a flow of fluid through a hollow cylinder, the velocity of that fluid has to increase at sites of narrowing along the cylinder for volume flow to be maintained. The volume flow is maintained by virtue of its dependence on the pressure gradient along the cylinder. The increase in velocity is proportional to the decrease in cross sectional area at the site of narrowing. The ratio of the velocity at the stenosis to the velocity before the stenosis (velocity ratio) equals the ratio of the area before the stenosis to the area at the stenosis. ${ }^{13}$ This area ratio is proportional to the amount of lumenal area lost as the result of the constriction or stenosis and is therefore related to the degree of stenosis (fig 2). Increasing the amount of stenosis will increase the velocity ratio. This principle is applied to assess the degree of stenosis, with Doppler ultrasound, in carotid arteries with atherosclerosis. ${ }^{10}{ }^{12}$ The velocity ratios for carotid arteries have been validated with angiographic studies.

An analogous situation can be seen in the PDA. The initial site of narrowing of the ductus arteriosus is most commonly at the pulmonary end. ${ }^{14}$ Therefore the ratio of velocity at the pulmonary end of the duct to the aortic end (the TVR) will be proportional to the degree of constriction of the PDA. The greater the degree of constriction, the less the left to right shunt through the PDA and its haemodynamic effect. Our hypothesis was that the TVR would be lower in infants with a haemodynamically significant PDA. We are not aware of any studies investigating the use of the TVR in assessing the significance of PDAs.

Various echocardiographic criteria have been used to assess the haemodynamic significance 


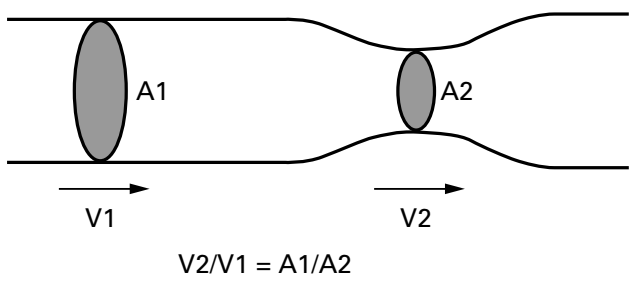

Figure 2 Relation between velocity of fluid flow $(V)$ and cross sectional area $(A)$ in a hollow cylinder.

of a PDA and the need for specific treatment. The haemodynamic significance will depend on the amount of left to right shunt across the PDA. The consequences of the shunt include increased left ventricular output, left heart volume overload, and reversal or absence of aortic diastolic flow distal to the duct. The most reliable indicators of the haemodynamic significance of a PDA are an increased LA/Ao ratio $^{115}{ }^{16}$ and reverse aortic diastolic flow. ${ }^{17-19}$ Other criteria include a pulsatile pattern of flow in the PDA, ${ }^{11}$ a wide ductal colour Doppler flow jet (at its narrowest point), ${ }^{19-21}$ wide smallest internal duct diameter measured on two dimensional imaging, ${ }^{22}$ and an increased LVIDd. $^{23}{ }^{24}$ Almost all the above criteria were assessed in our unit during the period of the study, which allowed comparison with the TVR. Ductal diameter was not routinely measured during this period; however, a subjective assessment of whether the duct was wide open or not was made, if possible, on two dimensional imaging.

The TVR was significantly less in those infants with a haemodynamically significant PDA, as assessed by individual echocardiographic criteria, in all groups except for those with dilatation of the left ventricle. This difference in TVR is even greater if the two most significant criteria (increased LA/Ao and reverse/absent diastolic aortic flow) are combined. Indices of left heart dilatation were increased in the group of infants with low TVR ( $<60 \%$ constriction), and there was a trend towards this group having more infants with reverse/absent diastolic aortic flow. These data confirm our hypothesis that, as the duct constricts and the left to right shunt decreases, the TVR increases. Conversely, the wider open the duct, the lower the TVR and the greater the left to right ductal shunt.

There are disadvantages to using the TVR to assess ductal significance. The technique depends on obtaining views of the PDA through an adequate acoustic window and this is not always possible, especially in the ventilated infant. Doppler signals may not be available from both ends of the PDA. The technique depends on a relatively straight ductus, and is therefore unhelpful if the duct is long and tortuous. As with any Doppler technique, the angle of insonation is important, and it is critical that the angle of insonation to blood flow is the same at both ends when measuring the PSV. The TVR cannot be used if there is right to left ductal flow and is therefore unhelpful in this circumstance. This would most commonly limit the usefulness of this technique in the first few days of life. If the TVR can be calculated, then a decreased TVR probably still represents a significant duct even in the first few days of life; however, we need to study more infants to confirm this.

There are times when adequate acoustic windows do not allow visualisation of other cardiac structures or the PDA on two dimensional imaging, but a Doppler signal can still be obtained from the duct. Calculation of the TVR can therefore be especially useful in instances when the ductal diameter cannot be measured.

We believe the TVR can be a useful adjunct to the other echocardiographic features seen with a PDA. After birth, the ductus arteriosus usually constricts and then closes. In well infants, almost all ducts are closed, at least functionally, by the fourth day of life. ${ }^{25}$ This may take considerably longer in ill, small, or preterm infants. ${ }^{26}{ }^{27}$ The pattern of closure of the ductus is quite variable in preterm infants. ${ }^{19}$ Closure may be delayed beyond the usual first few days of life, but there is still the possibility of spontaneous closure. ${ }^{19}$ It is our conjecture that evidence on echocardiography of constriction (increased TVR), despite other evidence of haemodynamically significant left to right shunting-for example, increased LA/Ao and/or reverse/absent diastolic aortic flow-may allow specific treatment (particularly surgery) to be delayed to await spontaneous closure. This should be accompanied by frequent clinical and echocardiographic review. In contrast, a low TVR signifying the absence of constriction may prompt early treatment of a PDA in the absence of other evidence of a significant left to right shunt. This use of the TVR to predict closure or likely continuing patency of a PDA has not been studied and further research is required.

CONCLUSION

The TVR as a measure of the degree of constriction of a PDA is associated with other echocardiographic criteria for a haemodynamically significant PDA. A low TVR signifying a poorly constricted duct is associated with other established echocardiographic features of a significant left to right shunt, and vice versa. Further research is required to determine the usefulness of the TVR in predicting closure or likely continuing patency of a PDA and the need for treatment.

\footnotetext{
1 Evans N. Diagnosis of patent ductus arteriosus in the preterm newborn. Arch Dis Child 1993;68:58-61.

2 Shimada S, Kasai T, Konishi M, Fujiwara T. Effects of patShimada S, Kasai T, Konishi M, Fujiwara T. Effects of pat-
ent ductus arteriosus on left ventricular output and organ blood flows in preterm infants with respiratory distress syndrome treated with surfactant. F Pediatr 1994;125:270-

3 Lindner W, Seidel M, Versmold HT, Dohlemann C, Riegel KP. Stoke volume and left ventricular output in preterm infants with patent ductus arteriosus. Pediatr Res 1990;27:278-81.

4 Evans N, Moorcraft J. Effect of patency of the ductus arteriosus on blood pressure in very preterm infants. Arch Dis Child 1992;67:1169-73.

5 Evans N, Kluckow M. Early ductal shunting and intraventricular haemorrhage in ventilated preterm infants. Arch Dis Child 1996;75:F183-6.

6 Cassady G, Crouse DT, Kirklin JW, et al. A randomized, controlled trial of very early prophylactic ligation of the ductus arteriosus in babies who weighed $1000 \mathrm{~g}$ or less at birth. N Engl f Med 1989;320:1511-16.

7 Brown ER. Increased risk of bronchpulmonary dysplasia in infants with patent ductus arteriosus. $f$ Pediatr 1979;95:865-6.
} 
8 Dudell GG, Gersony WM. Patent ductus arteriosus in neonates with severe respiratory distress. I Pediat $15-20$

9 Sidhu PS, Allan PL. Ultrasound assessment of internal carotid artery stenosis. Clin Radiol 1997;52:654-8.

10 Zwiebel WJ. New doppler parameters for carotid stenosis. Semin Ultrasound CT MR 1997;18:66-71.

11 Su B, Watanabe T, Shimizu M, Yanagisawa M. Echocardiographic assessment of patent ductus arteriosus shunt flow pattern in premature infants. Arch Dis Child Fetal Neonatal Ed 1997;77:F36-40.

12 Bluth EI, Stavros AT, Marich KW, Wetzner SM, Aufrichtig D, Baker JD. Carotid duplex sonography: a multicenter recommendation for standardized imaging and doppler criteria. Radiographics 1988;8:487-506.

13 Burns PN. Haemodynamics: a Doppler primer. Australasian Society for Ultrasound in Medicine Bulletin 1998;1:5-17.

14 Hammerman C. Patent ductus arteriosus. Clinical relevance of prostaglandins and prostaglandin inhibitors in PDA
pathophysiology and treatment. Clin Perinatol pathophysiology

15 Phillopos EZ, Robertson MA, Byrne PJ. Serial assessment of ductus arteriosus hemodynamics in hyaline membrane disease. Pediatrics 1996;98:1149-53.

16 Iyer P, Evans N. Re-evaluation of the left atrial to aortic root ratio as a marker of patent ductus arteriosus. Arch Dis Child Fetal Neonatal Ed 1999;70:F112-17.

17 Serwer GA, Armstrong BE, Anderson PAW. Continuous wave doppler ultrasonographic quantitation of patent ductus arteriosus flow. F Pediatr 1982;100:297-9.

18 Ulmer HE, Knapp G, Wolf D, Wille L, Seyberth HW. Aortic flow velocity curves in the diagnosis and the followup of symptomatic patent ductus arteriosus in preterm infants during therapeutic interventions. Pediatric Pharmacology 1983;3:167-74

19 Evans N, Iyer P. Longitudinal changes in the diameter of the ductus arteriosus in ventilated preterm infants: correlation with respiratory outcomes. Arch Dis Child Fetal Neonatal Ed 1995;72:F156-61.

20 Roberson DA, Silverman NH. Color doppler flow mapping of the patent ductus arteriosus in very low birthweight neonates: echocardiographic and clinical findings. Pediatric Cardiology 1994;15:219-24.

21 Kluckow M, Evans N. Early echocardiographic prediction of symptomatic patent ductus arteriosus in preterm infants undergoing mechanical ventilation. $f$ Pediatr 1995;127:774-9.

22 Sahn DJ, Allen HD. Real-time cross-sectional echocardiographic imaging and measurement of the patent ductus 1978;58:343-54

23 Baylen B, Meyer RA, Korfhagen J, Benzing G, Bubb ME, Kaplan S. Left ventricular performance in the critically ill premature infant with patent ductus arteriosus and pulmonary disease. Circulation 1977;55:182-8.

24 Hirschklau MJ, DiSessa TG, Higgins CB, Friedman WF. Echocardiographic diagnosis: pitfalls in the premature infant with a large patent ductus arteriosus. F Pediatr 1978;92:474-7.

25 Reller MD, Ziegler ML, Rice MJ, Solin RC, McDonald RW. Duration of ductal shunting in healthy preterm infants: an echocardiographc color flow doppler study. 7 Pediatr 1988;112:441-6.

26 McKone RC, Weesner KM. Determination of the time of closure of the ductus arteriosus in severely ill premature infants. Clin Pediatr 1988;27:135-9.

27 Desligneres S, Larroche JC. Ductus arteriosus. I. Anatomical and histological study of its development during the second half of gestation and its closure after birth. II. Histological study of a few cases of patent ductus arteriosus in infancy. Biol Neonate 1970;16:278-96. 